\title{
THE TREATMENT OF EPILEPSY BY SODIUM-DI-PHENYL HYDANTIONATE (EPANUTIN).
}

\author{
By DONALD BLAIR, M.A., M.B., Ch.B.(Cantab). D.P.M.(Lond.). \\ (Assistant Medical Officer, Cane Hill Mental Hospital.)
}

The frequent and ubiquitous occurrence of epilepsy renders it a disease of general interest throughout the medical profession. There are few, if any, diseases which are of such interest to practitioner and specialist alike. The idiopathic epileptic is the ever-present problem for the general practitioner and the neurologist; the epileptic with mental deficiency or psychosis is equally prominent in the experience of the psychiatrist; while specialists in other lines, when confronted with epileptics, always have to bear in mind the effect that any treatment they may advise will have on epilepsy apart from the more immediate disease, and frequently modify it accordingly.

Unfortunately, our knowledge of the pathology and ætiology of this disease is 'still so meagre as to make the treatment of its causation virtually impossible (except, of course, where it occurs secondarily to such organic diseases as brain tumours or paresis). The advent of exhaustive biochemical investigations and the introduction of electrical recordings of the brain rhythm bid fair to throw a new light on the whole subject, but in the meantime, the welfare of epileptic patients depends more on empirical treatment by anti-convulsant drugs than anything else.

The control of epileptic fits by the various anti-convulsants has met with only limited success. For each in turn great claims have been vaunted and great hopes held out, only to be followed later by comparative disillusionment. Ideally, each drug should exhibit the maximum of anti-convulsant properties with the minimum of toxic properties; the better known drugs used in the control of epilepsy leave much to be desired in this respect. In these circumstances, the introduction into the therapeutic field of such efficacious anti-convulsant properties as those claimed for sodium-di-phenyl hydantionate is bound to catch the eye of the medical world. Although only in its trial stages, protagonists and antagonists already abound, and erroneous ideas of its possibilities and its dangers are liable to spread. It therefore behoves the physician who wishes to administer this drug to study its properties impartially before embarking on its use.

\section{Sodium-di-phenyl Hydantionate.}

Dissatisfaction with the therapeutic agents already used for the treatment of epilepsy led Merritt and Putnam (I938) to experiment in search of new and better drugs of this type. Producing epileptic seizures in animals by means of electrical stimulation of the brain with the skull intact (i.e. with the electrodes applied to the exterior of the skull) they investigated a large number of compounds. They found that sodium-di-phenyl hydantionate stood out as having relatively the greatest anti-convulsant and least sedative effects; and in the latter respect it was much superior to luminal (phenobarbitone). They followed this by trying the drug out on human beings, and published the results of the treatment of a group of 200 epileptics in the Journal of the American Medical Association in September, I938. 
They stated that this hydantionate " was effective in controlling convulsive seizures in a great majority of a selected group of patients who were not helped by other methods of therapy." The selection excluded all cases other than those who had been subject to fits for many years and obtained little or no benefit from the usual treatments (bromides and the barbiturates). At the same time the authors had to admit that it was " without doubt considerably more toxic than bromides and the barbituric acid compounds." Since then the drug has been put on the market commercially as "Dilantin " in the United States and as "Epanutin" in this country.

Beyond various letters and commentaries, no work concerning the extensive clinical trial of this drug had been forthcoming until quite recently. Papers by the following authors have now appeared:-Blair, Bailey and MacGregor (I939) dealing with 75 cases, Steel and Seymour Smith (I939) with 20 cases, MacCartan (I939) with 20 cases and Frost (I939) with I2. All these authors tend to confirm Merritt and Putnam's findings regarding the strong anti-convulsant properties and marked toxic effects of this drug, while at the same time adding various observations of their own.

\section{Anti-convulsant properties.}

Merritt and Putnam's group of 200 cases, included cases of grand mal, petit mal and psychic equivalents, alone and in various combinations. Of these, I42 had received treatment for from two to twelve months with the following results : Grand mal fits, present in II8 cases, were completely relieved (i.e. total cessation of fits) in 58 per cent., greatly decreased in 27 per cent., moderately decreased in 4 per cent. and unchanged in 12 per cent. These figures are remarkable, but it must be noted that the exact period for which each case was completely or partially relieved is not given, nor do the authors define what is meant by " greatly decreased " and "moderately decreased." Moreover, in view of the findings of others, the fact that there were no cases with an increased number of fits seems unusual. They do instance two individual cases, however, which illustrate the dramatic possibilities of this drug. These patients had each been having fits daily for a considerable period before the new treatment (one of them on as much as 6 grs. of phenobarbitone daily), but were completely relieved for periods of as long as seven months and nine months respectively on being put on to " Dilantin." Another patient, suffering from petit mal and on $6 \mathrm{grs}$. of luminal (phenobarbitone) daily with little effect, was completely relieved for as long as three months following the introduction of "Dilantin."

Blair's, Bailey's and MacGregor's cases were all psychotic epileptics in mental hospitals and therefore under continuous observation. Of the 75 in their series, 52 had been on continuous treatment for over three months, and 6 for over two months; in II cases it had been necessary to terminate treatment owing to serious toxic effects, and the other 6 recent cases had not yet received treatment for two months. There was no selection beyond the exclusion of those so aged or feeble that a change from their usual sedative was not advisable. The figures of results were computed in relation to grand mal fits only. From a collective point of view they represent their results for the 58 cases of over three months' continuous 
treatment in comparison with Merritt's and Putnam's cases of grand mal only, in the following table:-

Effects of "Epanutin" on grand mal compared with results of Merritt and Putnam.

$\begin{array}{ccc}\text { Effect on frequency of } & \text { Merritt and Putnam's } & \text { Present } \\ \text { attack of grand mal. } & \text { series. } & \text { serics. }\end{array}$

\begin{tabular}{|c|c|c|c|c|c|}
\hline Complete relief & $\ldots$ & $\ldots$ & $\ldots$ & $29(49.1 \%)$ & $23(39.6 \%)$ \\
\hline Great decrease & $\ldots$ & $\ldots$ & $\ldots$ & $14(23.7 \%)$ & $12(20.6 \%)$ \\
\hline Moderate decrease & & $\ldots$ & $\ldots$ & $6(10.1 \%)$ & $5(8.6 \%)$ \\
\hline No change & $\ldots$ & $\ldots$ & $\ldots$ & $10(16.9 \%)$ & $4(6.8 \%)$ \\
\hline Increase $\quad \ldots$ & $\ldots$ & $\ldots$ & $\ldots$ & Not mentioned & $14(24.1 \%)$ \\
\hline Total & $\ldots$ & $\ldots$ & $\ldots$ & 59 & 58 \\
\hline
\end{tabular}

Merritt and Putnam do not state exactly the meaning of their terms; we have adopted the following criteria :-

Complete relief ... ... No fits during the last 6 weeks.

Great decrease ... ... Reduction by more than a half ... compared with a similar

Moderate decrease $\quad .$. Any reduction smaller than a half period before the treatment.

They also give a table of the number of fits occurring in each of the 58 caseso individually during their period on "Epanutin" compared with those during the corresponding periods (date for date) in the three previous years and the period of similar duration immediately prior to the "Epanutin" treatment. This table shows the true state of things from the point of view of the individual cases. The results make it clear that the effect of the anti-convulsant properties was in certain cases little less dramatic than those of Merritt and Putnam. Amongst these were such reductions as from 256 to $7 I, I 23$ to 9 , and I3I to 8 fits. On the other hand, there was one case in which an increase from IO2 to 221 fits occurred.

Steel and Seymour Smith's (I939) material consists of 20 cases of varying degrees of chronicity of between one and ten years. They found that six (30 per cent.) cases had a considerable diminution in number of fits, six (30 per cent.) a slight diminution, five (25 per cent.) no change, and three (I5 per cent.) an increase. They again instanced a few cases of an outstanding reduction of fits.

The papers of MacCartan and Frost, broadly speaking, confirm those already mentioned concerning the anti-convulsant effect on the majority of epileptics and more especially on certain individuals who seem to be particularly susceptible to its action and benefit almost miraculously. Reports on individual cases of the latter type have also been made by Denis Williams (I939) and Coope (I939). Nevertheless, final conclusions can only be attained when related to certain outstanding facts which must always be borne in mind when trying to reach a true verdict.

I. It is well known that chronic epileptics when changed over from one drug to another tend to stage a momentary benefit, sometimes of considerable duration and magnitude, only to revert subsequently to their previous condition. 
2. A prolonged cessation of fits in epileptics who have been habitually addicted to them may not be as beneficial as appears on the surface. For instance, it has been noticed that on such occasions when a convulsion does occur it may be much more severe than the normal ones. Steel and Smith also make this observation, and Denis Williams has informed me (personal communication) that certain of his cases felt the increased effects of such ultimate convulsions to such an extent that they preferred to have the more frequent and less severe fits.

3. Cases who receive complete relief from fits for as long as several weeks on end and might be placed in the completely relieved group, cannot necessarily be said to be going to benefit ultimately, as I have seen three cases who have pursued such a course for from four to six weeks suddenly have a batch of from I5 to 20 fits in three or four days. Steel and Smith report several "disappointing relapses." It is even possible that a case might go one step further and end in status epilepticus.

4. In certain cases showing a decrease of major seizures there is an increase in minor ones (petit mal).

5. Certain unfavourable mental symptoms, such as confusion (see below), may result from a prolonged freedom from fits.

\section{Mental effects.}

Merritt and Putnam stressed the practically complete absence of sedative effect in "Epanutin." This is of great importance, since one of the chief drawbacks of luminal (phenobarbitone) in many cases, is that when given in sufficient quantities to control the fits, it has also a soporific and almost stupefying effect. Blair, Bailey and MacGregor instanced a case who " in a state of semi-stupor on gr. $\frac{1}{2}$ of luminal thrice daily, had his stuporose condition completely relieved by Epanutin (I capsule t.d.s.)." They also state that the majority of their cases have shown temperamental benefit, a matter of no small consequence to those dealing with the psychotic cases. MacCartan's results tend to confirm this.

It must not be thought however that all cases are affected favourably. Certain cases have followed the reverse process and become mentally more troublesome. One must be apprehensive of undue optimism in the early stages, since one case showed complete cessation of fits for several weeks, associated with a simultaneous very obvious improvement in his temperament; but about eight weeks after the commencement of treatment, the patient entered into a state of prolonged confusion; this was similar to that he usually experienced before a fit, but on this occasion no fit was forthcoming and the confusion continued for six weeks on end, more prolonged and severe than he had ever had before. At the end of this period "Epanutin" capsules were reduced by one per diem and the patient had a fit soon after and recovered from the confusion in his usual manner. Here again, therefore, we have an example of a case " completely relieved " whose condition was really worse than before.

\section{Toxic effects.}

The great disadvantage of this drug is its toxicity. Its anti-convulsant effects on some patients are striking, but toxic effects may be equally so. Merritt and Putnam in their original article described the following toxic symptoms:-

(I) Slight giddiness, ataxia, tremors, blurring of vision and diplopia in I5 per cent. of their cases, occurring from the 3 rd to the Ioth day. 
(2) Several minor skin rashes of an erythematous, scarletiniform or morbilliform nature.

(3) One case of exfoliative dermatitis and one of purpura occurring on the 9 th and roth days.

In view of the experiences of others, these toxic possibilities must be considerably enlarged upon, and may now be listed as follows:-

Nervous symptoms.

Dizziness, giddiness, blurring of vision, diplopia, clonic spasms, tremors, general muscular irritability, ataxia and nystagmus.

Mental symptoms.

Euphoria, excitement, agitation, irritability, confusion, delusions and hallucinations, dullness, depression, and reactivation of suicidal tendencies.

\section{Skin eruptions.}

Mostly of a mild erythematous, scarletiniform or morbilliform type, but sometimes of a more severe nature accompanied by pyrexia and very occasionally of such severity as to be an exfoliative dermatitis.

Gastro-intestinal symptoms.

Nausea, anorexia, abdominal discomfort, constipation, etc.

Sponginess and tenderness of the gums.

Other symptoms.

Epistaxis (Frost, I939), slight blood changes of varying and unconclusiveo descriptions.

Although these frequently occur soon after the introduction of "Epanutin" or a rise in its dosage, this is by no means always the case, especially regarding the mental symptoms which may occur weeks or even months after the patient has been on "Epanutin."

The gastro-intestinal symptoms are due to the alkalinity of the drug and are almost invariably relieved by giving it with a drink of water during or just after a meal. Blood changes of such a nature as leucopenia, lymphocytosis eosinophilia and an increase in hæmoglobin percentage have been recorded, but no change of a serious nature has yet been seen. The incidence of sponginess and tenderness of the gums was originally described as present in a large percentage of cases (Kimball, I939) but has only occasionally been seen by other writers.

\section{Treatment of Toxic Symptoms.}

The above makes a formidable array of mishaps, but fortunately, provided that their presence is recognised as due to the drug, an immediate and efficacious remedy is always at hand, namely, complete withdrawal of the drug. When this step is taken, the disappearance of the symptoms is usually almost as rapid as their onset. Occasionally, however, they take days and even weeks to clear up finally. and this is so particularly after a rise in dosage, or when there has been undue delay in withholding the drug. The best procedure in all such cases would seem to be immediate and complete cessation of the drug without its replacement by other anti-convulsants. If the toxic symptoms clear up within a few days, "Epanutin" treatment may be started again. If three capsules a day were being given, a 
similar number may be tried again, but if more than three, reintroduction should always commence with one less capsule per diem. In the event of an excessive occurrence of fits following cessation of "Epanutin" (no such case has so far been recorded), immediate resort should be made to intramuscular injection of luminal followed by a return to the sedative used before "Epanutin" was started. Contra-indications to a return to "Epanutin " are (i) those cases with exceptionally severe toxic symptoms; (ii) those who, after being on "Epanutin" for a reasonable length of time, have fared no better regarding the number of fits than they did on previous medications. It may be added that in certain cases the abovementioned nervous and mental symptoms may reach unpleasant and disabling severity, especially if the drug is not stopped on the first toxic indications. For instance, three of my cases developed ataxia following an increase of dosage of from three to four capsules a day in one case and from four to five capsules in the other two. The drug was purposely not stopped at once as I wished to test whether the symptom was momentary and would cease with a rapid increase of tolerance. This was not the case and within two days of the increased dosage the ataxia had in each case assumed such proportions that the patients were unable to stand up without support, or even when supported reeled about in a manner reminiscent of very severe alcoholic or cerebellar tumour symptoms. It is also worthy of mention that liability of toxic symptoms increase with dosage.

\section{Dosage.}

One interesting fact regarding dosage is that in all adults a minimum of .3 gms. per day (3 capsules) seems to be a necessity, regardless of how small a dose of sedative the patient was on before. I have had opportunity to confirm this in two cases who had been free from fits for more than a year on tab. luminal grs. I $\frac{1}{2}$ once a day and gr. I nocte respectively. They were both put on to one "Epanutin" capsule twice a day. A few weeks later they each had several fits over the course of a few days. The dosage was increased to one capsule three times a day, with no subsequent fits up to date.

Blair, Bailey and MacGregor point out the lack of correlation between the dosage of "Epanutin" required to control the fits and that of the previous sedative. They stress the importance of giving one of the capsules just before going to bed in those subject to nocturnal fits, a procedure which makes all the difference to control of this type of case. They investigated thoroughly the changeover from previous sedatives to "Epanutin" and advise the following method of substitution: if the patient is previously on three doses of sedative, replace one dose (regardless of its quantity) by one capsule of "Epanutin " every alternate day. Thus by the end of the sixth day the patient will be on "Epanutin " only. If the previous sedative is being given four times daily, withdraw the fourth dose on the 8th day without replacing it by an "Epanutin" capsule. "Epanutin" is not increased beyond one capsule t.d.s. until after the I4th day, unless there is a marked increase in fits, as it is during this time that toxic symptoms are most frequent.

\section{Conclusions.}

According to our present knowledge, the following conclusions regarding the action of "Epanutin " seem tentatively to be warranted:-

(I) A minimum threshold dosage of .3 gms. a day (three capsules) is essential in all adult cases. Neglect to give this sufficiency, no matter 
how small the dosage of previous sedative, will almost certainly lead to fits.

(2) The action of the drug is rapid and does not appear to be cumulative.

(3) Early withdrawal in toxic cases has almost immediate effect.

(4) It has a specific effect in each individual case, and in many only produces really effective anti-convulsant action when given in such a dosage as to cause toxic symptoms.

(5) There are certain cases in which its anti-convulsant action apparently predominates, and who have been on it for many months without any toxic manifestations.

(6) Some cases appear to acquire an increased tolerance, as exemplified by the absence of toxic symptoms on the occasions of its reintroduction. Others appear to become more susceptible, as witness those who develop late toxic symptoms.

(7) The mode of its action is uncertain. It seems to be of the nature of an increased resistance to the convulsive discharge. In many cases this leads to a condenser effect, with the result that fits, although less frequent, are more severe; while in others, following a prolonged cessation of fits there is a sudden outburst of many fits in a short time.

(8) We are far from a complete understanding of its actions, and a finab judgment must await more prolonged and extensive trial.

Application. Pending a final verdict regarding the therapeutic potentialities of this drug, or the discovery of a derivative of equal anti-convulsant and less toxie properties, its use would appear indicated under the following conditions in cases failing to benefit from the better known and less toxic anti-convulsants :-

(a) Provided that the toxic symptoms are fully appreciated and such precautions taken as may ensure the patient's being seen at the earliest possible moment following these appearances and the drug immediately stopped.

(b) Provided that facilities for hospital treatment are available if necessary.

(c) In psychotic cases with marked temperamental defects.

Finally, as this drug's paramount vindication, one may stress certain cases in which its anti-convulsant properties are very marked and its toxic properties are absent, and others whose temperamental reactions are improved, although the reduction of fits is slight. These advantages would appear to give "Epanutin " a definite place in the therapy of epilepsy, provided that indiscriminate use is avoided and adequate precautions taken. At the same time, more prolonged and extensive observation is required before a final verdict regarding its therapeutic potentialities is pronounced.

REFERENCES.

BLAIR, DONALD, BAILEY, K. C. and MAcGREGOR, J. S. (1939), The Lancet, 2, 363.

COOPER, R. (1939), The Lancet, $1,180$.

KIMBALL, O. P. (1939), Jour. Amer. Med. Assocn., April 1, p. 1244.

MCCARTAN, W. (1939) and FROST, I. (1939) read Papers on "Epanutin" treatment at the Royal Medico-Psychological Association's Annual Meeting at Brighton in July, 1939, to be published in print in the Journal of Mental Science,

September, 1939 .
MERRITT, H. H. and PUTNAM, T. J. (1938), Archiv. Neurol. \& Psychiat., 39, 1003 ; Jour. Amer. Med. Assocn., $111,1068$. STEEL, J. P. and SMITH, E. S. (1939), The Lancet, $2,367$.

WILLIAMS, DENIS (1939), Proc. Roy. Soc. Med., 32,Vol. xxxii. 872. 